\title{
Case-control study of infections with Salmonella enteritidis phage type 4 in England
}

\author{
J M Cowden, D Lynch, C A Joseph, M O’Mahony, S L Mawer, B Rowe, C L R Bartlett
}

\begin{abstract}
Objective-To determine the source of indigenous sporadic infection with Salmonella enteritidis phage type 4.

Design-Case-control study of primary sporadic cases identified by the Public Health Laboratory Service between 1 August and 30 September 1988.

Setting-PHLS Communicable Disease Surveillance Centre, Division of Enteric Pathogens, 11 PHLS laboratories, and 42 local authority environmental health departments in England.

Subjects-232 Patients (cases) with confirmed primary sporadic infection, for 160 of whom $(88$ female) (median age 30 years, age range 4 months to 85 years) data were obtained by questionnaire about consumption of fresh eggs, egg products, precooked chicken, and minced meat in the three days and one week before onset of the symptoms. Up to three controls, matched for neighbourhood, age, and sex (if aged $>11$ years), were asked the same questions for the same calendar period.
\end{abstract}

Main outcome measure-Association of primary sporadic infection with consumption of suspected food items.

Results-Illness due to $S$ enteritidis phage type 4 was significantly associated with consumption of raw shell egg products (homemade mayonnaise, ice cream, and milk drinks containing eggs) (matched $\mathbf{p}=0.02$ ) and shop bought sandwiches containing mayonnaise (matched $p=0.00004$ ) or eggs (matched $\mathbf{p}=\mathbf{0 . 0 2}$ ). Illness was also significantly associated with eating lightly cooked eggs (unmatched $p=0.02$ ), but not soft boiled eggs, and precooked hot chicken (matched $\mathbf{p}=\mathbf{0} \cdot 006$ ). Reported consumption of eggs was not appreciably different between cases and controls before or after the median date of interview.

Conclusions-Fresh shell eggs, egg products, and precooked hot chicken are vehicles of $\boldsymbol{S}$ enteritidis phage type 4 infection in indigenous sporadic cases. Public health education and reduction in contamination of eggs and infection of poultry with $S$ enteritidis are needed to reduce the incidence of human infection.

Disease Surveillance

Centre, London NW9 5EQ

$\mathrm{J} M$ Cowden, $\mathrm{MB}$, senior registrar

D Lynch, BSC, statistician C A Joseph, MSC, assistant epidemiologist

M O'Mahony, MFCM, consultant epidemiologist

C L R Bartlett, FFCM, director

PHLS Laboratory, Hull

S L Mawer, FRCPATH, director

Division of Enteric Pathogens, Central Public Health Laboratory

B Rowe, FRCPATH, director

Correspondence to: $\mathrm{Dr}$ Cowden.

BrMed F 1989;299:771-3 identified eggs, chicken, and minced meat, including hamburgers. We tested the hypothesis that these foods were associated with sporadic infection with this organism with a case-control study.

\section{Subjects and methods}

After a standard questionnaire had been developed in a pilot study the case-control study was carried out by the Communicable Disease Surveillance Centre, the Division of Enteric Pathogens, 11 PHLS laboratories, and 42 local authority environmental health departments throughout England. The laboratories were selected to give a wide geographical spread and to include rural and urban areas.

A primary sporadic case was defined as a patient from whom $S$ enteritidis phage type 4 was isolated from faecal culture between 1 August and 30 September 1988 , who was the first or only person in the household to have had gastrointestinal symptoms, who had not returned from domestic or foreign travel in the week before the illness, or who was not part of a point source outbreak. Three controls, who had not been ill in the four weeks before the patient who nominated them, were matched for neighbourhood (to a maximum of $8 \mathrm{~km}$ ), age, and sex (if over 11 years old). If a control in the neighbourhood was not nominated or could not be contacted other strategies were acceptable. At least one appropriate control was interviewed for each case. The questionnaire sought information on symptoms, consumption of fresh shell eggs or raw or cooked foods containing them, chicken, and minced meat in the three days and the week before the onset of symptoms. Patients and controls were asked the same questions for the same calendar period.

Unless otherwise stated statistical analysis was carried out with an exact one tailed probability test, taking into account variable matched groups.' The null hypothesis for matched analysis was that in the cases consumption of the food item in the three days before the onset of symptoms was no more likely than in controls.

\section{Results}

Altogether, of 232 patients eligible for the study, 160 $(69 \%)$ were interviewed, 72 of whom were male and 88 female. Their ages ranged from 4 months to 85 years (median 30 years); 34 were aged under 10 years and 13 over 65. Altogether, 157 patients reported diarrhoea, 132 abdominal pain, 116 fever, and 92 nausea. Illness lasted from under one day to over one month (median seven days). Thirty two patients were admitted to hospital for between one and 11 days (median five days).

One, two, or three controls were obtained for each of the 157 cases. Of the 196 controls, 132 were nominated from the neighbourhood; 43 from work, school, or social group; seven by general practitioners; six were contacted by randomly knocking on doors; and in the remaining eight the interviewer did not specify the source. The date of interview of 117 of the 150 subjects for whom it was recorded was within three weeks after the onset of illness. The median interval between onset of illness and interview was 14 days.

The table shows the five independent associations that were observed between infection and eating various food items in the three days before the onset of 
Consumption of food items in cases of $S$ enteritidis phage tvpe 4 infection and controls. Figures are numbers (percentages)

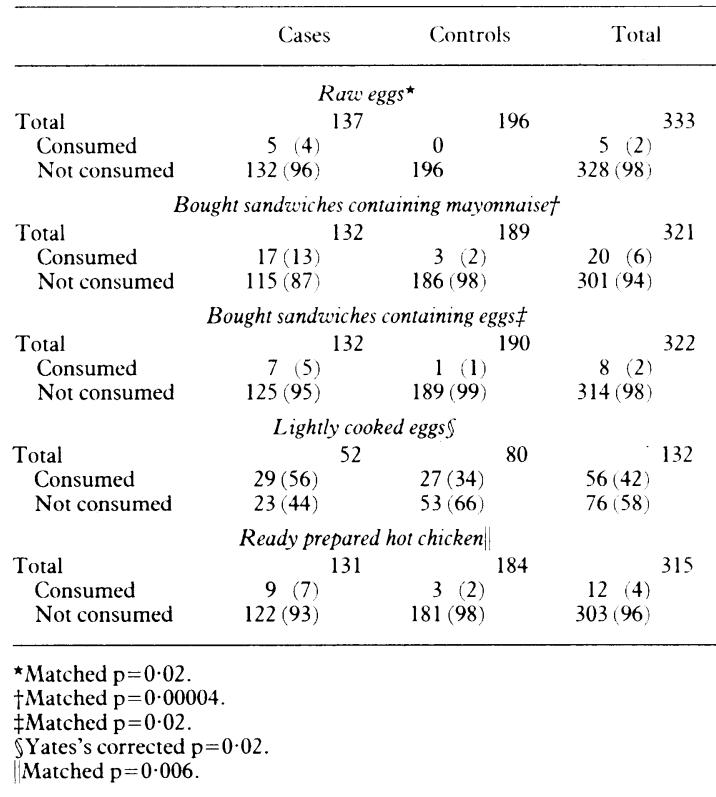

symptoms. Eating raw shell egg products (homemade mayonnaise, ice cream, and milk drinks containing raw shell eggs) at home was significantly associated with illness (matched $p=0 \cdot 02$ ). Eating shop bought sandwiches containing mayonnaise or eggs was signifcantly associated with illness (matched $\mathrm{p}=0.00004$, $0 \cdot 02$, respectively). Of 137 patients, $26(19 \%)$ ate at least one of the products specified in the table compared with only $4(0.02 \%)$ of 196 controls.

Among the 132 people who ate eggs scrambled, in an omelette, fried, or poached, illness was associated on unmatched analysis with eating runny eggs (table). The difference in consumption of soft boiled eggs in 14 cases $(10 \%)$ and 27 controls (14\%) was not significant. Eating ready prepared hot chicken (for example, a take away dish) was also significantly associated with illness (matched $\mathrm{p}=0.006$ ).

Although the consumption of eggs and egg products listed in the questionnaire was not different in the cases and controls, the controls were more likely to have eaten at least one of the meat products - namely, chicken and minced meat including hamburgers ( 158 controls $(81 \%)$ $v 91(66 \%)$ cases Yates's corrected $\left.\gamma^{2}=8.96, \mathrm{p}=0.003\right)$.

Reported egg consumption was not significantly different among subjects interviewed before and after 7 September, the median date of interview.

\section{Discussion}

This study implicated eggs, egg products, and ready prepared hot chicken as vehicles of infection in indigenous sporadic cases of illness due to $S$ enteritidis phage type 4. Studies of sporadic cases are important as the vehicles that cause them need not necessarily be responsible for outbreaks. The findings do not stand in isolation. Hens' eggs have been known to cause salmonellosis for many years ${ }^{2}{ }^{3}$ but recent outbreaks of $S$ enteritidis infection have been linked with intact fresh shell eggs and their products in the United Kingdom and elsewhere (PHLS Communicable Disease Surveillance Centre, unpublished data).$^{+-6}$

The association of illness with eating eggs and products containing eggs is consistent and supports evidence from veterinary and microbiological studies, from analytical studies of outbreaks in this country and abroad, and from another recent case-control study of sporadic cases. ${ }^{7}$ Although the response rate in this study was lower than the $81 \%$ achieved in one recent investigation of salmonellosis, ${ }^{8}$ response rates of $63 \%$ and $72 \%$ have been sufficient to show associations in other studies. ${ }^{4}$ Most interviewers were environmental health officers, who make every effort to contact all patients with food poisoning in their districts, and though insufficient data are available on the subjects who were not interviewed to infer possible bias, such bias is unlikely as the age and sex distributions in our cases were similar to those in cases of salmonella infection reported to the surveillance centre (PHLS Communicable Disease Surveillance Centre, unpublished data). As response rates from all laboratories were similar it is unlikely that any geographical bias was introduced. In the study nominated controls in the neighbourhood were included, a method of selection shown to be effective in recent national outbreaks of salmonellosis in England (unpublished observations). ${ }^{8}$ Systematic bias was probably not introduced by the interviewers' knowledge of the hypotheses being tested; lightly boiled eggs were not associated with illness, as might have been expected had bias occurred. The strength of the association with shop bought sandwiches (especially containing mayonnaise) was also surprising.

None of the raw egg products implicated was commercially prepared, and widespread poor practice in domestic kitchens is unlikely. Although cross contamination might have led to the association with shop bought egg sandwiches, other possibilities exist; in boiling large numbers of eggs, it may be difficult to achieve thorough cooking of them all that is necessary. ${ }^{10-12}$ The strength of the association with any shop bought sandwich containing mayonnaise is striking. Questions about the composition and method of preparation of the mayonnaise were not asked. The possibility cannot be excluded that mayonnaise produced in bulk, perhaps using pasteurised liquid egg, either on or off the premises of shops selling sandwiches, was the vehicle of infection.

There was no overall association between eating lightly cooked eggs and illness; this could be explained by low rates of contamination of eggs, and by other vehicles leading to human infection. All lightly cooked egg dishes associated with illness require breaking the eggs out of their shells before cooking. Matched analysis was not possible for these dishes because too few groups contained both a patient and a control who were sure that they had or had not eaten eggs cooked in this way.

External contamination of the egg shell might explain all the findings of this study, including the association of illness with eating egg sandwiches, which may be explained by cross contamination. Transovarian infections with $S$ enteritidis phage type 4 have, however, been reported in a broiler breeder flock, ${ }^{13}$ and the organism was found in the ovaries and oviducts of hens from a laying flock in which the infection was prevalent. ${ }^{14}$

The association between illness and eating ready prepared hot and take away chicken is not surprising. Poultry meat remains an important source of salmonella infections in humans, ${ }^{15}$ and recent work has shown $S$ enteritidis phage type 4 to be the commonest type in fresh and frozen chicken on retail shelves. ${ }^{16} \mathrm{It}$ is therefore surprising that the controls were more likely than cases to have eaten poultry, other than hot ready prepared chicken. The controls, however, were also more likely to have eaten other meats; non-meat eaters may tend to eat more eggs and dairy produce, and meat eating may therefore have a spurious protective effect.

In only $63(39 \%)$ of 160 cases was consumption of at least one of the implicated food vehicles in the three days before onset of symptoms reported; in 15 additional cases one of these had been consumed between three and seven days before. Perhaps raw or lightly cooked egg consumed unknowingly, poor recall, or the existence of other food vehicles contributed to this finding. 
Publicity linking eggs with salmonellosis probably did not affect the results. Much of the press coverage referred to the situation in the United States. That there was no significant difference between reported egg consumption in subjects interviewed before or after the median date of interview implies that neither government statements or media coverage influenced people in their reporting.

England and Wales are experiencing an epidemic of food poisoning caused by $S$ enteritidis phage type 4 . This study supports previous findings that eggs, egg products, and precooked chicken are significant vehicles of infection and for the first time in a large national study confirms their importance in indigenous sporadic cases. The proportion of eggs contaminated is low, ${ }^{16}$ but because as many as $\mathbf{3 0}$ million eggs are consumed daily the number of human infections caused nationally represents an important public health problem. Whether imperfect practice in kitchens has contributed to the striking increase in $S$ enteritidis food poisoning or not there is no evidence that standards have declined in recent years; the best solution is to combine public health education with a reduction in contamination of eggs and infection of poultry with salmonella. This will require the eradication of $S$ enteritidis from layer and broiler flocks, and the first stage in this strategy must be to identify infected flocks.

We thank the directors and staff of the following PHLS laboratories for their contribution: Ashford, Bristol, Cambridge, Guildford, Exeter, Hull, Newcastle upon Tyne,
Nottingham, Preston, Taunton, and Wolverhampton; the medical officers for environmental health, infection control nurses, and environmental health officers who made such a valuable contribution; and Dr P G Smith for his advice in preparing the manuscript.

1 Tillett HE. Statistical analysis of case-control studies of communicable diseases. Int $\mathcal{O}$ Epidemiol 1986;15:126-33.

2 Scott WM. Food poisoning due to eggs. Br Med f 1930; ii:56-9.

3 Broomhead CL, Mann PE. A family outbreak of Salmonella thompson infection. Monthly Bulletin of the Ministry of Health and Public Healt Laboratory Service 1959;18:124-7.

4 St Louis ME, Morse DL, Potter ME, et al. The emergence of grade A eggs as a major source of Salmonella enteritidis infections. $\mathcal{F} A M A$ 1988;259:2103-7.

5 Feng-Ying CL, Morris JE, Trump D. Investigation of an outbreak of Salmonella enteritidis gastroenteritis associated with consumption of eggs in a restaurant chain in Maryland. Am $\mathcal{F}$ Epidemiol 1988;128:839-44.

6 Perales I, Audicana A. Salmonella enteritidis and eggs. Lancet 1988;ii:1133.

7 Coyle EF, Palmer SR, Ribeiro CD, et al. Salmonella enteritidis phage type 4 infection: association with hens' eggs. Lancet 1988;ii:1295-6.

8 Cowden JM, O'Mahy Salm J $O$ 'Mahony, Epidemiol Infect (in press)

9 Mitchell E, O'Mahony M, Lynch D, et al. Large outbreak of food poisonin caused by Salmonella typhimurium definitive type 49 in mayonnaise. Br.Med f 1989;298:99-101.

10 Humphrey TJ, Greenwood M, Gilbert RJ, Rowe B, Chapman PA. The survival of salmonellas in shell eggs cooked under simulated domestic conditions. Epidemiol Infect (in press).

11 Licciondello JJ, Nickerson JTR, Goldblith SA. Destruction of salmonellae in hard boiled eggs. Am f Public Health 1965;55:1622-8.

12 Baker RC, Hogarty S, Poon W, Vadehra DV. Survival of Salmonell typhimurium and Staphylococcus aureus in eggs cooked by different methods. Poult Sci 1983;62:1211-6.

13 Lister SA. Salmonella enteritidis infection in broilers and broiler breeders. Vet Rec 1988:123:350.

14 Hopper SA, Mawer S. Salmonella enteritidis in a commercial layer flock. Yet Rec 1988:123:351.

15 Humphrey TJ, Mead GC, Rowe B. Poultry meat as a source of human salmonellosis in England and Wales. Epidemiol Infect 1988;100:175-84 16 Anonymous. Public Health Laboratory Service Microbiologv Digest 1989;6:1-9.

Accepted 21 fuly 1989
Department of Virology, United Medical and Dental Schools of Guy's and St Thomas's Hospitals, St Thomas's Campus, London SE1 7EH

J M Best, PHD, reader J E Banatvala, FRCPATH, professor

Department of Virology, Preston Infirmary, Preston PR1 6PS

P Morgan-Capner,

MRCPATH,

consultant virologist

PHLS Communicable Disease Surveillance Centre, London NW9 5EQ $\mathrm{E}$ Miller, $\mathrm{MB}$, top grade epidemiologist

Correspondence to: Dr Best.

Br.Med F 1989;299:773-5

\title{
Fetal infection after maternal reinfection with rubella: criteria for defining reinfection
}

\author{
J M Best, J E Banatvala, P Morgan-Capner, E Miller
}

\section{Abstract}

Five cases of asymptomatic maternal reinfection with rubella are described that occurred in England and Wales during 1985-8 and resulted in intrauterine infection. The criteria for diagnosing reinfection are described. In four cases the rubella contact was with the woman's own children. Two women had therapeutic abortions, rubella virus being recovered from the products of conception, and three were delivered of infants with congenitally acquired disease.

Though the risks associated with maternal reinfection with rubella are very small and being measured in a prospective study, it is hoped that the recently introduced augmented programme of rubella vaccination will reduce rubella in the community and therefore this small risk still further.

\section{Introduction}

Reinfection with rubella may occur and has been reported after both naturally acquired and vaccine induced infection. Reinfection is usually subclinical and is detected serologically, most commonly among pregnant women who have had close and prolonged contact with rubella at home. Reinfection in pregnancy has been considered to present a minimal risk to the fetus, and mothers are usually reassured that there is no risk or only a minimal one to the fetus. ${ }^{12}$ Nevertheless, there have been several isolated reports of fetal infection and malformation resulting from maternal re- infection (reviewed by Morgan-Capner ${ }^{3}$ ). We report five such cases.

\section{Patients and methods}

Cases were referred by obstetricians, paediatricians, and microbiologists or were identified from reports to the Communicable Disease Surveillance Centre by laboratories in England and Wales during 1985-8 Standard techniques were used for serological testing of mothers and infants and for isolating rubella virus from the products of conception or from throat swabs taken from infants. ${ }^{+}$When possible the avidity of specific IgGl was measured; high avidity suggests recent reinfection.

\section{Results}

The table shows details of the five cases; all five women were without symptoms throughout pregnancy. Three women (cases 1-3) were serologically investigated after contact with rubella between four and eight weeks' gestation, when their children had symptoms like rubella. IgM antibody (specific for rubella) was detected in serum samples taken from all three women after contact; subsequent samples from two of them (cases 1 and 2) showed a decline in titre. Reinfection was diagnosed because antibody had been detected by radial haemolysis at $>15000 \mathrm{IU} / 1$ on two occasions before the affected pregnancy; these serum samples were not available for retesting. The results of 